\title{
Induced Membrane Technique Combined with a Free Vascularized Fibula Flap for the Management of a Severe Acute Femoral Bone Loss: A Case Report and Literature Review
}

\author{
Xu-sheng Qiu, PhD ${ }^{1}$ Xin Zheng, $\mathrm{PhD}^{1} \quad$ Hong-fei Shi, $\mathrm{PhD}^{1} \quad$ Guang-yue Xu, MD ${ }^{1} \quad$ Yi-xin Chen, MD, $\mathrm{PhD}^{1}$ \\ ${ }^{1}$ Department of Orthopaedics, Drum Tower Hospital, the Affiliated \\ Hospital of Nanjing University Medical School, Nanjing, Jiangsu, \\ China \\ Address for correspondence Yi-xin Chen, MD, PhD, Department of \\ Orthopaedics, Drum Tower Hospital, the Affiliated Hospital of Nanjing \\ University Medical School, No. 321 Zhongshan Road, Nanjing, Jiangsu \\ 210008, China (e-mail: chenyixin93@126.com).
}

J Reconstr Microsurg Open 2017;2:e15-e18.

\begin{abstract}
Keywords

- induced membrane technique

- segmental bone defects

- free vascularized fibular flap

Background Management of segmental bone defects is a challenge for orthopedic surgeons. The most commonly used methods are the free vascularized bone transfer, bone transport, and induced membrane technique. However, none of these methods is fully suitable for some cases.

Case Report A 41-year-old woman was presented with a Gustilo IIIA left distal femoral fracture (AO 32-C and 33-C) with bone loss. The length of femoral bone loss was $15 \mathrm{~cm}$ (12 cm medially and $18 \mathrm{~cm}$ laterally). After thorough debridement, the intercondylar fracture was fixed with K-wires, and an external fixator bridged the knee joint. The bone defect was filled with antibiotic cement spacer. After 2 months, the external fixator was removed; the femur was fixed by internal fixation. The induced membrane was opened and the antibiotic cement removed. A free vascularized fibular graft of $18 \mathrm{~cm}$ was transferred and the residual bony defect was filled with morcellized cancellous autologous bone graft. At 6 months postoperatively, the graft appeared completely integrated at X-ray and the patient could walk without brace at 11 months postoperatively.

Conclusion The distal femur is a large-caliber bone and is under severe stress during weight bearing. When only the induced membrane technique is used, the autologous bone graft seems not enough for the present case. On the other hand, single free vascularized fibular graft also could not provide sufficient strength to allow weight bearing despite significant hypertrophy. Therefore, the induced membrane technique combined with free vascularized fibular graft used in the present case may be an alternative.
\end{abstract}

The management of bone defects, especially segmental bone defects, is a challenge for orthopedic surgeons. The most commonly used methods for the reconstruction of segmental bone defects are the free vascularized fibula flap, Ilizarov bone transport technique, and induced membrane techni- que. ${ }^{1-3}$ Every method has its own shortcomings. ${ }^{1-3}$ The combination of these methods may overcome their shortcomings. However, there were few reports on the combined use of these methods. ${ }^{4}$ The present case report describes a case of severe acute femoral bone loss managed by an induced received

June 16, 2015

accepted after revision

December 5, 2016
DOI http://dx.doi.org/

10.1055/s-0037-1598045. ISSN 2377-0813.
Copyright $\odot 2017$ by Thieme Medical Publishers, Inc., 333 Seventh Avenue, New York, NY 10001, USA. Tel: +1(212) 584-4662.
License terms

(c) $(1) \ominus$ 


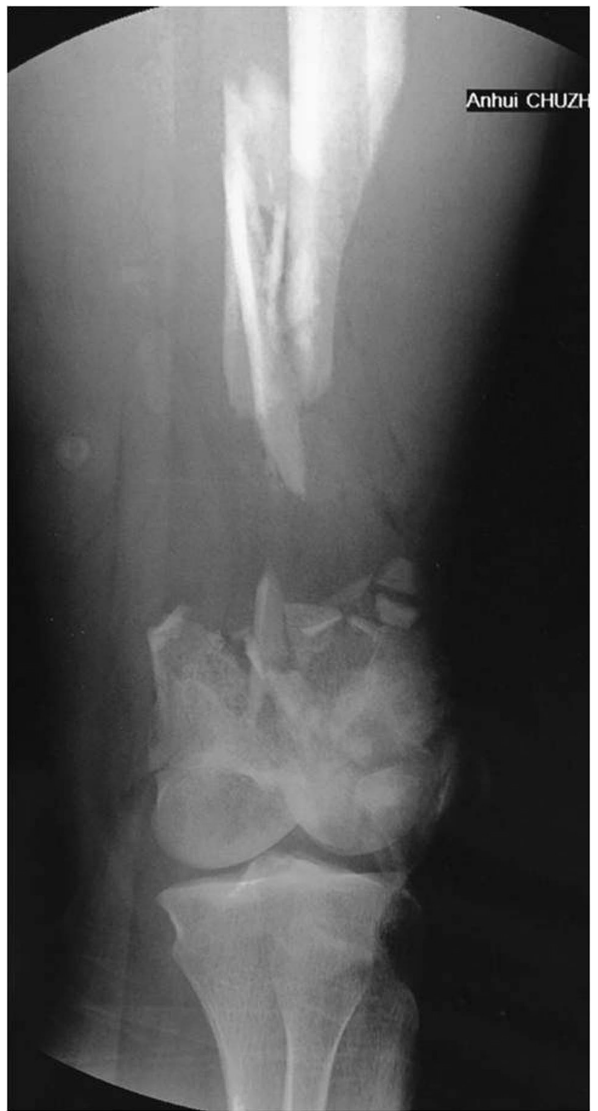

Fig. 1 A 41-year-old woman was admitted with a Gustilo IIIA left femur meta-epiphyseal fracture (AO 32-C and 33-C) and left patellar fracture. The length of femoral bone loss was $15 \mathrm{~cm}(12 \mathrm{~cm}$ medially and $18 \mathrm{~cm}$ laterally). membrane technique combined with a free vascularized fibula flap.

\section{Case Report}

A 41-year-old woman was admitted after a motorcycle accident. The patient presented with a Gustilo IIIA left femur meta-epiphyseal fracture (AO 32-C and 33-C) and left patellar fracture (-Fig. 1). The length of femoral bone loss was $15 \mathrm{~cm}$ (12 cm medially and $18 \mathrm{~cm}$ laterally).

Thorough debridement was performed. Tissue samples were collected for microbiological culture. The intercondylar fracture was fixed with K-wires, and an external fixator bridged the knee joint (Hoffman II external fixation system, Stryker, Kalamazoo, MI). The bone defect was then filled with antibiotic cement spacer, which was premixed with gentamycin (Smith \& Nephew, Memphis, TN) (-Fig. 2). The patellar fracture was fixed with two screws. The open wound was primarily closed.

Intravenous antibiotic therapy with vancomycin $500 \mathrm{mg}$ $\times 4$ daily was started immediately postoperatively. Weight bearing was not allowed postoperatively. The microbiological cultures were negative and the wound healed uneventfully. Antibiotic therapy was discontinued after 1 week, and the patient was discharged after 9 days.

Blood examinations (erythrocyte sedimentation rate [ESR], C-reactive protein [CRP], and white blood cell [WBC] count) were checked routinely during follow-up and normalized after 1 month. The external fixator was removed and the limb was braced in a cast 1.5 months after the initial procedure. The second step of the Masquelet technique was performed 2 months after the trauma.
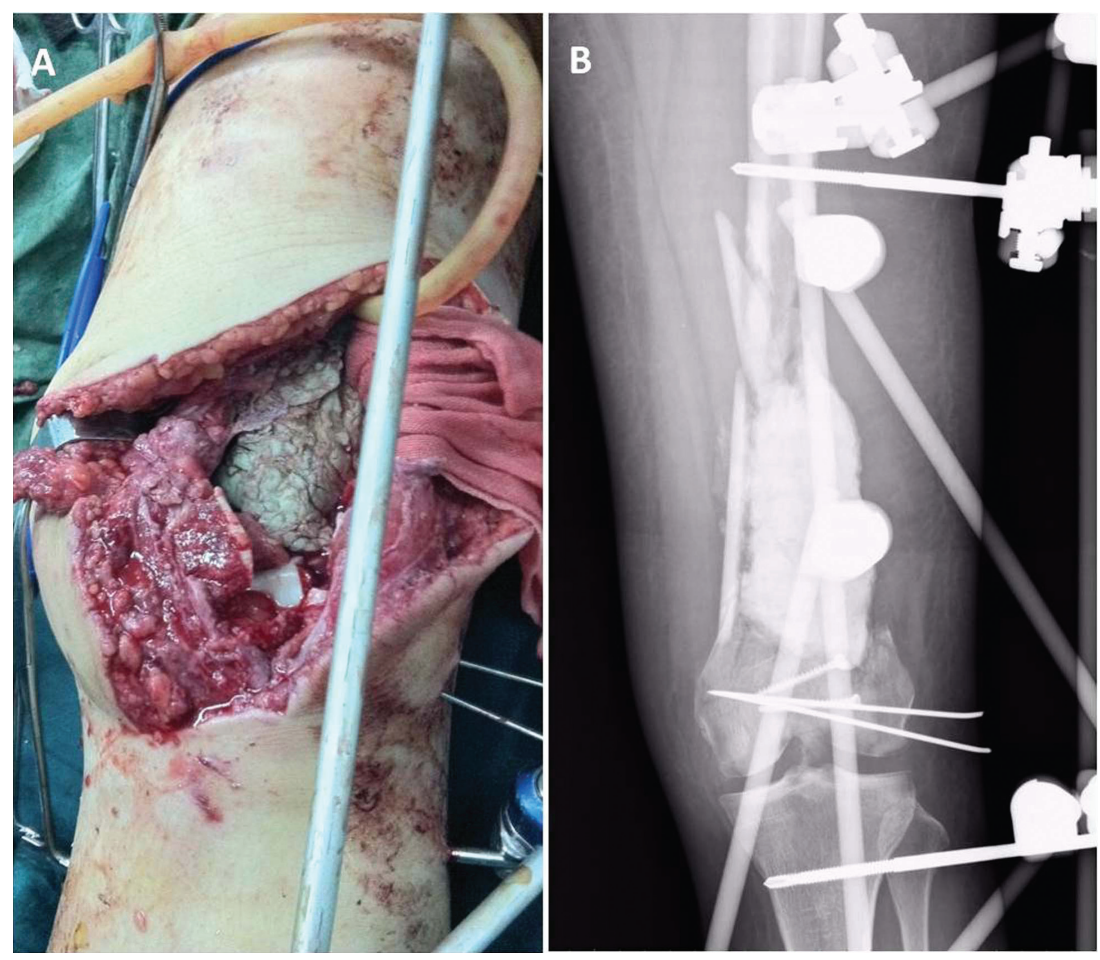

Fig. 2 First step of the Masquelet technique. (A) The femoral bone defect was filled with a cement spacer. (B) X-ray of the cement spacer in an anteroposterior view. Proper alignment and length of the limb obtained with external fixation. 


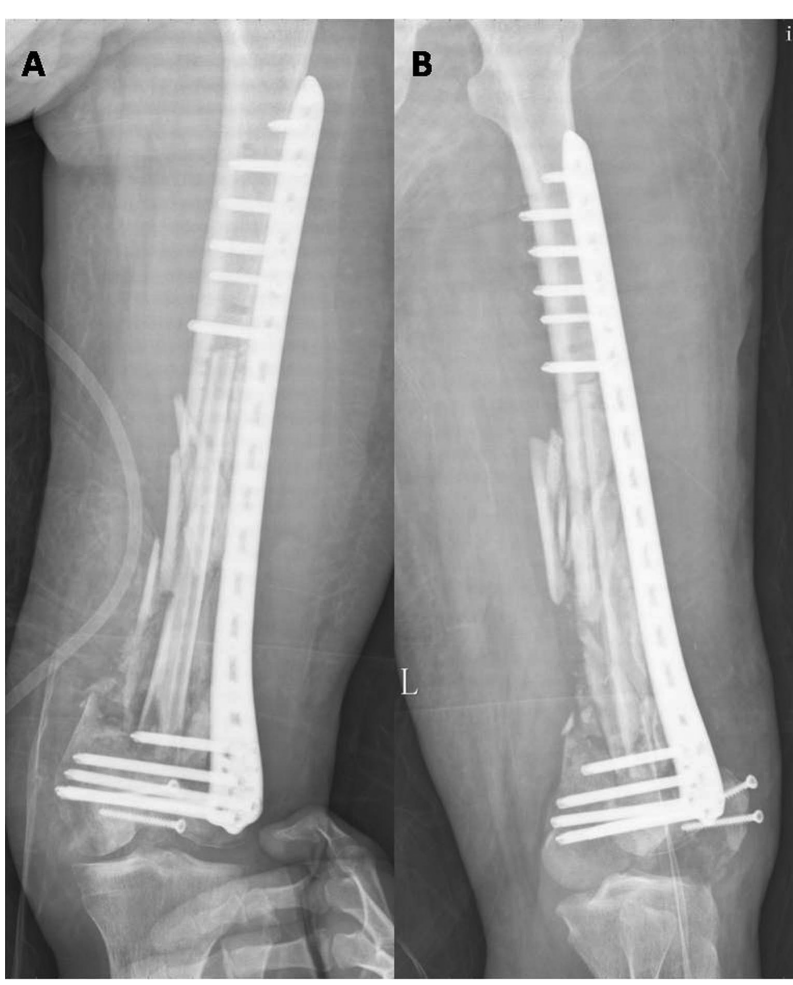

Fig. 3 X-ray showed the composite graft and plate in an anteroposterior (A) and lateral (B) views. A complete filling of the defect was obtained, maintaining correct limb alignment and length.
A lateral approach to the femur was used. The induced membrane was opened and the antibiotic cement was removed. Samples for microbiological culture were collected. A free vascularized fibula $(18 \mathrm{~cm})$ was harvested from the contralateral leg. The donor peroneal artery was anastomosed to the branch of the femoral artery by the end-toend technique. The peroneal veins were anastomosed to the venae comitantes of the recipient artery and the saphenous vein. The residual bony defect was filled with morcellized cancellous autologous bone graft from iliac crests. Then, the induced membrane was closed. The femur was fixed by internal fixation with the less invasive stabilization system (LISS) (-Fig. 3). A superficial drainage was placed and subcutaneous and skin layers were sutured.

One week after surgery, antibiotic therapy (vancomycin $500 \mathrm{mg} \times 4$ daily) was discontinued on the basis of negative microbiological cultures. Weight bearing was not allowed for the first 2 months. Partial weight bearing started at 3 months, was progressive at 4 months, and full at 5 months. At 6 months, the graft appeared completely integrated on the $\mathrm{X}$-ray (-Fig. 4). It was interesting that significant hypertrophy of the transferred fibula was not found at 3 years followup (-Fig. 4). We thought that the hypertrophy of the fibula was not needed because the fibula had a surrounding consolidated morcellized cancellous autograft.

\section{Literature Review and Conclusion}

Between 1986 and 1999, Masquelet et al ${ }^{5,6}$ have developed the induced membrane technique, and 35 patients with

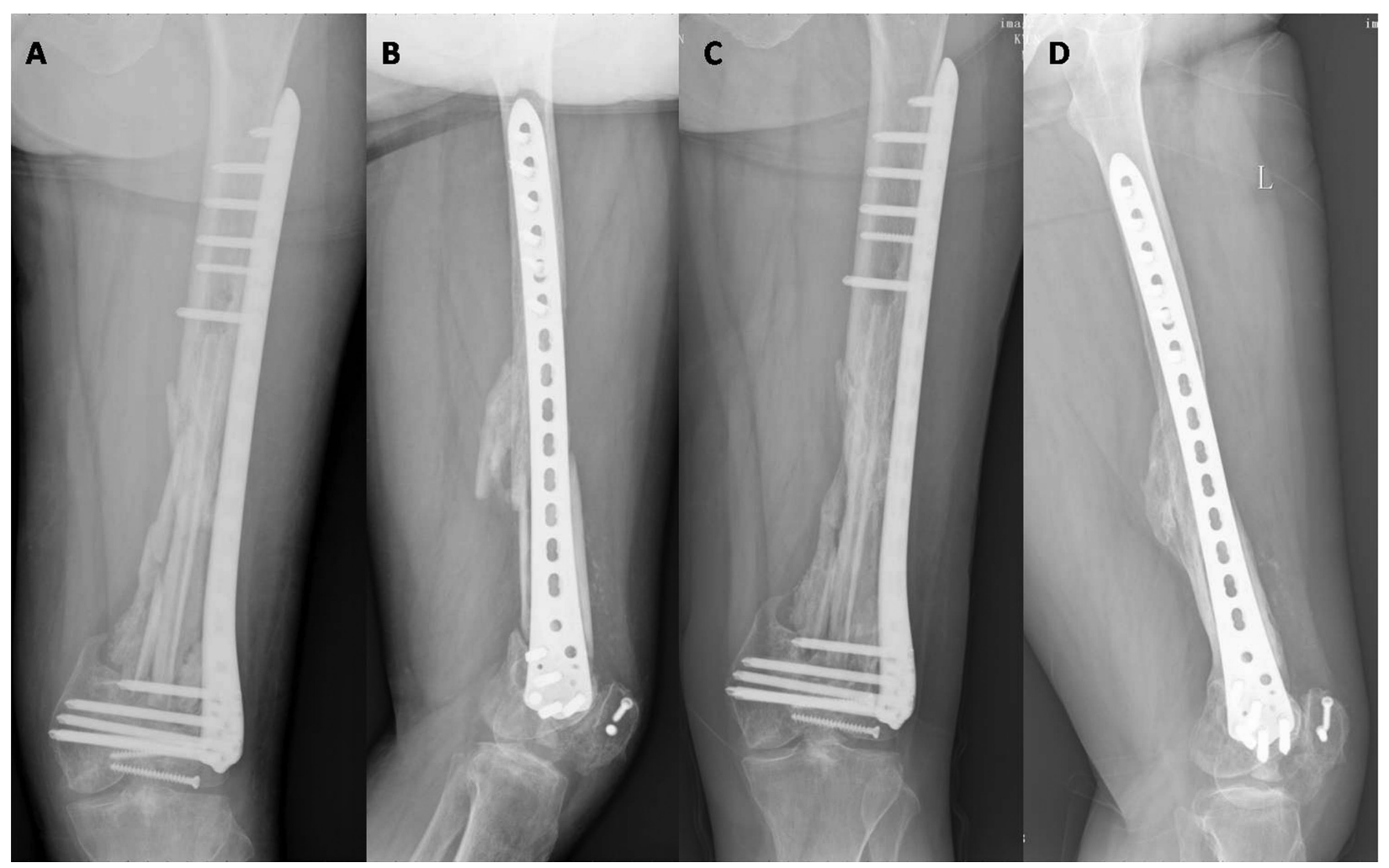

Fig. 4 X-ray follow-up. (A, B) Six months. Integration of the graft on both sides. (C, D) Three years. Remodeling of the morcellized cancellous autograft and hypertrophy of the transferred fibula were not significant because of the consolidation of the morcellized cancellous autograft. 
segmental bone defects were treated with the induced membrane technique. The bone defects ranged from 5 to $25 \mathrm{~cm}$. In 20 cases, the defect exceeded $10 \mathrm{~cm}$. Soft tissue repair by flaps was performed in 28 cases. Three patients failed due to the failures of free flaps, and bone healing was obtained by openair grafting or bone transport. One amputation was performed before bone healing. The other 31 patients, obtained bone healing at an average 4 months and normal walking without devices were allowed at an average of 8.5 months. Delayed stress fractures were noted in four patients, two at 6 months and two at 2 years. Healing was obtained by simple cast immobilization. All infections had healed without recurrence upon long-term follow-up.

The induced membrane technique was performed in two stages $^{3}$ : The first stage includes radical debridement and insertion of an antibiotic cement spacer into the bone defects. The bone was stabilized always with an external fixator. Soft tissue was repaired by flaps when needed. The second stage was performed 6 to 8 weeks later. The spacer was removed; a cancellous autograft was placed within this membrane. The induced membrane technique does not require specialized equipment; it can be performed easily by surgeons with various capabilities and experience; and it is applicable to diaphyseal, metaphyseal, or epiphyseal bone defects. Therefore, the induced membrane technique, as described by or modified from Masquelet, seems to have gained popularity recent years due to the management of segmental bone defects. $^{7-13}$

In the present case, the induced membrane technique was initially chosen for the management of segmental bone defects. However, for second-stage management, the autologous bone graft seemed insufficient due to the large metaepiphyseal bone defects in the femur. Additional grafting material was required. However, the ideal ratio of bone substitutes and autograft was not determined, although the ratio of $1: 3$ (allograft:autograft) was suggested. ${ }^{3}$ Therefore, the autologous bone graft combined with a free vascularized fibula flap was chosen in the present study.

The clinical outcomes of the vascularized free fibula flap for the management of large bone defects are favorable. ${ }^{2}$ However, the femur, especially the distal segment, is a large-caliber bone and is under severe stress during weight bearing. Significant hypertrophy must occur for the transferred fibula to be able to support the body weight. This hypertrophy can take a long period of time in adults and still may not provide sufficient strength to allow weight bearing. Therefore, the fracture of a single strut of fibula placed in the femur is common. ${ }^{14}$ An alternative is to use folded or double free vascularized fibula flaps. ${ }^{15}$ The third option for the femur is the free fibula used in conjunction with allografts. ${ }^{16}$ The induced membrane technique combined with the free vascularized fibula flap used in the present case may be a fourth option, or a modified method to a single free vascularized fibula flap to reconstruct segmental bone defects in the femur.

Pelissier et $\mathrm{al}^{17}$ demonstrated that the induced membranes have an abundant vascular network and secrete growth factors and osteoinductive factors. With the aforementioned characteristics, the membranous pocket prevents resorption of the contained graft and supports the revascularization and consolidation of the bone graft. Free vascularized fibula flap always has a reliable effect on the bone union because of its vessel-rich characteristics. ${ }^{2}$ Therefore, this combined method was reasonable as an alternative method for the reconstruction of segmental bone defects in femurs. The present case demonstrated that this combined method could be successfully used for the reconstruction of segmental femoral bone defects.

\section{References}

1 Aronson J. Limb-lengthening, skeletal reconstruction, and bone transport with the Ilizarov method. J Bone Joint Surg Am 1997; 79(08):1243-1258

2 Pederson WC, Person DW. Long bone reconstruction with vascularized bone grafts. Orthop Clin North Am 2007;38(01):23-35, v

3 Masquelet AC, Begue T. The concept of induced membrane for reconstruction of long bone defects. Orthop Clin North Am 2010; 41(01):27-37

4 Uzel AP, Lemonne F, Casoli V. Tibial segmental bone defect reconstruction by Ilizarov type bone transport in an induced membrane. Orthop Traumatol Surg Res 2010;96(02):194-198

5 Masquelet AC, Fitoussi F, Begue T, Muller GP. [Reconstruction of the long bones by the induced membrane and spongy autograft]. Ann Chir Plast Esthet 2000;45(03):346-353

6 Masquelet AC. Muscle reconstruction in reconstructive surgery: soft tissue repair and long bone reconstruction. Langenbecks Arch Surg 2003;388(05):344-346

7 Karger C, Kishi T, Schneider L, Fitoussi F, Masquelet AC; French Society of Orthopaedic Surgery and Traumatology (SoFCOT). Treatment of posttraumatic bone defects by the induced membrane technique. Orthop Traumatol Surg Res 2012;98(01):97-102

8 Apard T, Bigorre N, Cronier P, Duteille F, Bizot P, Massin P. Twostage reconstruction of post-traumatic segmental tibia bone loss with nailing. Orthop Traumatol Surg Res 2010;96(05):549-553

9 Stafford PR, Norris BL. Reamer-irrigator-aspirator bone graft and bi Masquelet technique for segmental bone defect nonunions: a review of 25 cases. Injury 2010;41(2, Suppl 2):S72-S77

10 Woon CY, Chong KW, Wong MK. Induced membranes-a staged technique of bone-grafting for segmental bone loss: a report of two cases and a literature review. J Bone Joint Surg Am 2010; 92(01):196-201

11 Giannoudis PV, Faour O, Goff T, Kanakaris N, Dimitriou R. Masquelet technique for the treatment of bone defects: tips-tricks and future directions. Injury 2011;42(06):591-598

12 Taylor BC, French BG, Fowler TT, Russell J, Poka A. Induced membrane technique for reconstruction to manage bone loss. J Am Acad Orthop Surg 2012;20(03):142-150

13 Mauffrey C, Hake ME, Chadayammuri V, Masquelet AC. Reconstruction of long bone infections using the induced membrane technique: tips and tricks. J Orthop Trauma 2016;30(06):e188-e193

14 Song HR, Kale A, Park HB, et al. Comparison of internal bone transport and vascularized fibular grafting for femoral bone defects. J Orthop Trauma 2003;17(03):203-211

15 Muramatsu K, Ihara K, Shigetomi M, Kawai S. Femoral reconstruction by single, folded or double free vascularised fibular grafts. Br J Plast Surg 2004;57(06):550-555

16 Chang DW, Weber KL. Use of a vascularized fibula bone flap and intercalary allograft for diaphyseal reconstruction after resection of primary extremity bone sarcomas. Plast Reconstr Surg 2005; 116(07):1918-1925

17 Pelissier P, Masquelet AC, Bareille R, Pelissier SM, Amedee J. Induced membranes secrete growth factors including vascular and osteoinductive factors and could stimulate bone regeneration. J Orthop Res 2004;22(01):73-79 